\title{
Clinical Course of Nodular Regenerative Hyperplasia in Thiopurine Treated Inflammatory Bowel Disease Patients
}

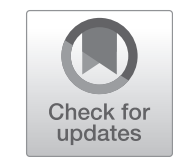

\author{
Melek Simsek, ${ }^{*, a}$ Berrie Meijer,, ${ }^{* a}$ Dewkoemar Ramsoekh, ${ }^{*}$ Gerd Bouma, ${ }^{*}$ \\ Egbert-Jan van der Wouden, ${ }^{\ddagger}$ Bert den Hartog, ${ }^{\S}$ Annemarie C. de Vries, ${ }^{\prime}$ Frank Hoentjen, ${ }^{\natural}$ \\ Gerard Dijkstra, ${ }^{\#}$ Sybrand Y. de Boer, ${ }^{\star \star}$ Jeroen M. Jansen, ${ }^{\neq \ddagger}$ Andrea E. van der Meulen, ${ }^{\S \S}$ \\ Ruud Beukers, "\| Menno A. Brink, ${ }^{\text {शा }}$ Toos Steinhauser," ${ }^{\# \#}$ Bas Oldenburg, ${ }^{* \star \star}$ \\ Lennard P. Gilissen, ${ }^{\ddagger \neq \ddagger}$ Ton H. Naber, ${ }^{\S \S \S}$ Marc A. Verhagen, ${ }^{\|\| \|}$Nanne K. H. de Boer, ${ }^{*}$ and \\ Chris J. J. Mulder, ${ }^{,}$on behalf of the Dutch Initiative on Crohn and Colitis (ICC)
}

\begin{abstract}
${ }^{*}$ Department of Gastroenterology and Hepatology, VU University Medical Center, Amsterdam, The Netherlands; ${ }^{\ddagger}$ Department of Gastroenterology and Hepatology, Isala Clinics, Zwolle, The Netherlands; ${ }^{\S}$ Department of Gastroenterology and Hepatology, Rijnstate Hospital, Arnhem, The Netherlands; "Department of Gastroenterology and Hepatology, Erasmus University Medical Center, Rotterdam, The Netherlands; "Department of Gastroenterology and Hepatology, Radboud University Medical Center, Nijmegen, The Netherlands; "Department of Gastroenterology and Hepatology, University Medical Center and University of Groningen, Groningen, The Netherlands; 'Department of Gastroenterology and Hepatology, Slingeland Hospital, Doetinchem, The Netherlands; ${ }^{\ddagger \pm}$ Department of Gastroenterology and Hepatology, Onze Lieve Vrouwe Gasthuis, Amsterdam, The Netherlands; ${ }^{\S}$ Department of Gastroenterology and Hepatology, Leiden University Medical Center, Leiden, The Netherlands; "IIDepartment of Gastroenterology and Hepatology, Albert Schweitzer Hospital, Dordrecht, The Netherlands; "19"Department of Gastroenterology and Hepatology, Meander Medical Center, Amersfoort, The Netherlands; "\#\#epartment of Gastroenterology and Hepatology, Bravis Hospital, Roosendaal, The Netherlands; ${ }^{* * *}$ Department of Gastroenterology and Hepatology, University Medical Center Utrecht, Utrecht, The Netherlands; ${ }^{\ddagger \neq}$ Department of Gastroenterology and Hepatology, Catharina Hospital,

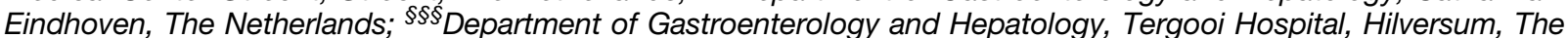
Netherlands; and "III"Department of Gastroenterology and Hepatology, Diakonessen Hospital, Utrecht, The Netherlands.
\end{abstract}

$\mathrm{N}$ odular regenerative hyperplasia $(\mathrm{NRH})$ is a poorly understood liver condition, which is increasingly recognized in thiopurine-treated patients with inflammatory bowel disease (IBD). ${ }^{1}$ It is difficult to establish an optimal approach to $\mathrm{NRH}$ patients, because its manifestations are highly variable (from asymptomatic to symptoms of noncirrhotic portal hypertension [NCPH]) and the prognosis is unknown. ${ }^{2}$ The aim of this study was to identify NRH cases in IBD patients treated with azathioprine, mercaptopurine, and/or thioguanine, and to describe its clinical course.

\section{Methods}

All 81 Dutch hospitals with a gastroenterology and hepatology department were requested to report NRH cases with detailed (follow-up) data. Collected data included demographics; disease and (historical) treatment characteristics; and clinical, laboratory, imaging, endoscopic, and histopathologic findings. Manifestations of NRH (signs or symptoms of NCPH or asymptomatic [ie, biochemical abnormalities in the absence of clinical signs of liver disease]) before diagnosis and during follow-up were documented. Solely cases of histopathologically proven NRH (grade 3 micronodularity in the absence of bridging fibrosis ${ }^{3}$ ) without concomitant liver pathology, as diagnosed by the pathologist at location, were included.

\section{Results}

Seventy-two hospitals (89\%) reported a total of 43 $\mathrm{NRH}$ cases in thiopurine-treated IBD patients. Their baseline characteristics are detailed in Table 1. Eighteen patients $(42 \%)$ were treated with thioguanine. Notably, almost $90 \%$ of them had previously received azathioprine and/or mercaptopurine. At time of NRH diagnosis, 17 patients (40\%) were asymptomatic, whereas 26 $(60 \%)$ presented with symptoms of $\mathrm{NCPH}$, being splenomegaly in $23(88 \%)$, ascites in $4(15 \%)$, and esophageal varices in 19 (73\%), with variceal bleeding in 11 patients (42\%). Patients with NCPH had lower hemoglobin $(7.3$ vs $8.5 \mathrm{mmol} / \mathrm{L} ; P<.05)$ and platelet count ( 90 vs $172 \times 10^{9} / \mathrm{L} ; P<.001$ ), but liver biochemistry did not differ between asymptomatic and NCPH patients.

After a median follow-up of 5.5 years (range, 2-13 years), signs and symptoms of NCPH resolved in 8 patients (31\%). Initially, NRH was complicated by thrombocytopenia and splenomegaly in all 8 patients; 6 patients had esophageal varices as well. In these 8 patients, aspartate aminotransferase (48 vs $30 \mathrm{U} / \mathrm{L}$ ) and

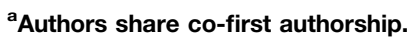

Most current article

(C) 2019 by the AGA Institute $1542-3565 / \$ 36.00$

https://doi.org/10.1016/j.cgh.2018.05.009 
Table 1. Baseline Characteristics, Liver Biochemistry, and Imaging at Time of Diagnosis and Follow-up $(n=43)$

\begin{tabular}{|c|c|c|}
\hline Sex, male & \multicolumn{2}{|l|}{$33(77)$} \\
\hline Age at $\mathrm{NRH}$ diagnosis, $y$ & \multicolumn{2}{|l|}{$49(17-74)$} \\
\hline IBD disease duration, $y$ & \multicolumn{2}{|l|}{$15(3-44)$} \\
\hline IBD (CD/UC/IBDu) ${ }^{a}$ & \multicolumn{2}{|c|}{$30(70) / 12(28) / 1(2)$} \\
\hline $\mathrm{CD}$ behavior (B1/B2/B3) & \multicolumn{2}{|c|}{$13(43) / 14(47) / 5(17)$} \\
\hline CD location (L1/L2/L3/L4) & \multicolumn{2}{|c|}{$14(47) / 6(20) / 8(27) / 2(7)$} \\
\hline UC extent (E1/E2/E3) & \multicolumn{2}{|c|}{$0(0) / 5(42) / 7(58)$} \\
\hline UC severity (S0/S1/S2/S3) & \multicolumn{2}{|c|}{$4(33) / 1(9) / 4(33) / 3(25)$} \\
\hline Thiopurine use (AZA/MP/TG) & \multicolumn{2}{|c|}{$30(70) / 13(30) / 18(42)$} \\
\hline AZA dose $(m g)$ and duration $(m o)$ & $150(100-300)$ & $48(1-204)$ \\
\hline MP dose $(m g)$ and duration $(m o)$ & $75(25-100)$ & $33(1-78)$ \\
\hline TG dose $(m g)$ and duration $(m o)$ & $21(20-48)$ & $38(12-84)$ \\
\hline Laboratory findings & At diagnosis & Follow-up \\
\hline $\mathrm{Hb}(7.5-11 \mathrm{mmol} / \mathrm{L})$ & $8.2(4.3-10.1)$ & $8.5(5.2-10.1)$ \\
\hline PC $\left(150-400 \times 10^{9} / \mathrm{L}\right)^{\star \star}$ & $115(43-346)$ & $160(28-319)$ \\
\hline $\operatorname{ALT}(<45 \mathrm{U} / \mathrm{L})^{\star \star \star}$ & $45(15-104)$ & $34(12-87)$ \\
\hline AST $(<40 \mathrm{U} / \mathrm{L})^{\star \star \star}$ & $44(18-78)$ & 30 (17-95) \\
\hline GGT $(<55 \mathrm{U} / \mathrm{L})^{\star \star \star}$ & $107(16-497)$ & 47 (12-272) \\
\hline $\operatorname{AP}(<120$ U/L) & $126(55-316)$ & $94(14-344)$ \\
\hline Bilirubin $(<20 \mu \mathrm{mol} / \mathrm{L})^{*}$ & $16(4-328)$ & $12(4-48)$ \\
\hline Imaging findings & At diagnosis & Follow-up \\
\hline No abnormalities* & $17(40)$ & $27(63)$ \\
\hline Splenomegaly* & $23(54)$ & $11(26)$ \\
\hline Heterogeneous parenchyma* & $22(51)$ & $13(30)$ \\
\hline Bleeding varices & $11(26)$ & $5(12)$ \\
\hline Ascites & $4(9)$ & $3(7)$ \\
\hline
\end{tabular}

NOTE. Categorical characteristics are depicted as numbers and percentages, continuous characteristics as medians with ranges.

Significance: ${ }^{*} P<.05 ;{ }^{\star \star} P<.01 ;{ }^{\star \star \star} P<.001$.

ALT, alanine aminotransferase; AP, alkaline phosphatase; AST, aspartate aminotransferase; AZA, azathioprine; CD, Crohn's disease; GGT, $\gamma$-glutamyltransferase; $\mathrm{Hb}$, hemoglobin; IBD, inflammatory bowel disease; MP, mercaptopurine; $\mathrm{NRH}$, nodular regenerative hyperplasia; PC, platelet count; TG, thioguanine; UC, ulcerative colitis.

${ }^{a}$ Disease-specific characteristics are depicted using the Montreal classification for $C D$ and UC.

alkaline phosphatase (127 vs $90 \mathrm{U} / \mathrm{L}$, both $P<.05)$ decreased, and there was a statistic trend toward a higher platelet count ( 99 vs $182 \times 10^{9} / \mathrm{L} ; P=.06$ ) during follow-up. Five patients (19\%) experienced recurrent variceal hemorrhages, of which 3 had a recurrence of ascites, despite secondary prophylaxis. All 5 patients had been exposed to azathioprine; 3 patients had been exposed to thioguanine as well.

The 17 patients without clinical signs of liver disease at diagnosis did not develop complications during followup. Overall, liver enzymes normalized and platelet count improved over time (Table 1). None of the patients received a liver transplantation, developed hepatocellular carcinoma, or died during the long-term course.

\section{Discussion}

The pathogenesis and prognosis of NRH are widely unknown, which makes it difficult to establish an optimal approach to patients with $\mathrm{NRH}^{4}{ }^{4}$ In this study, a relatively small number of $43 \mathrm{NRH}$ patients were identified in a nationwide cohort, of which 26 patients (60\%) presented with symptoms of NCPH at diagnosis. During a follow-up of 66 months, symptoms of NCPH resolved in 8 (31\%) and recurred in 5 patients (19\%). Both liver biochemistry and platelet count normalized in most patients.

Thiopurines are effective in maintaining remission in IBD, but concerns about adverse events, such as NRH, have impaired their use. Especially thioguanine, a thiopurine-derivative used as a rescue drug, has been related to hepatotoxicity, which has limited its use in IBD. ${ }^{5}$ In this cohort, $90 \%$ of thioguanine-exposed patients had previously used azathioprine and/or mercaptopurine. The causative factor of NRH in these cases, and in the available literature (incidence rates, 0\%-62\%), is questionable, because azathioprine and IBD itself have been related to NRH as well. 6 ,

Our cohort was characterized by a predominance of male sex and stricturing disease phenotype. Furthermore, we showed a significant reversibility of NCPH symptoms. In a systematic French study on NRH and azathioprine, NRH was mainly identified in male patients with stricturing Crohn's disease. ${ }^{6}$ In both their and our cohort, patients with asymptomatic $\mathrm{NRH}$ at time of diagnosis remained asymptomatic during extensive follow-up. Ferlitsch et $\mathrm{al}^{8}$ reported on improvement of portal pressure in thiopurine-exposed NRH patients, after the cessation of thiopurines. In our study, portal venous pressure was not measured, but we observed reversibility of NRH-related complications (especially in young patients), indicative for portal pressure decrease.

In conclusion, in the long-term follow-up of $43 \mathrm{NRH}$ patients, predominantly male with (stricturing) Crohn's disease, liver biochemistry and platelet count normalized after thiopurine discontinuation. Symptoms of NCPH resolved in one-third and recurred in 5 patients (with pre-existing severe $\mathrm{NCPH}$ at diagnosis).

\section{References}

1. Musumba CO. Review article: the association between nodular regenerative hyperplasia, inflammatory bowel disease and thiopurine therapy. Aliment Pharmacol Ther 2013;38:1025-1037.

2. Al-Mukhaizeem KA, Rosenberg A, Sherker AH. Nodular regenerative hyperplasia of the liver: an under-recognized cause of portal hypertension in hematological disorders. Am J Hematol 2004;75:225-230.

3. Wanless IR. Micronodular transformation (nodular regenerative hyperplasia) of the liver: a report of 64 cases among 2,500 autopsies and a new classification of benign hepatocellular nodules. Hepatology 1990;11:787-797.

4. Hartleb M, Gutkowski K, Milkiewicz P. Nodular regenerative hyperplasia: evolving concepts on underdiagnosed cause of portal hypertension. World J Gastroenterol 2011;17:1400-1409.

5. Taylor KM, Ward MG, Blaker PA, et al. Is there a role for thioguanine therapy in IBD in 2017 and beyond? Expert Rev Gastroenterol Hepatol 2017;11:473-486.

6. Vernier-Massouille G, Cosnes J, Lemann M, et al. Nodular regenerative hyperplasia in patients with inflammatory bowel disease treated with azathioprine. Gut 2007;56:1404-1409.

7. De Boer NK, Tuynman H, Bloemena E, et al. Histopathology of liver biopsies from a thiopurine-naive inflammatory bowel 
disease cohort: prevalence of nodular regenerative hyperplasia. Scand J Gastroenterol 2008;43:604-608.

8. Ferlitsch A, Teml A, Reinisch W, et al. 6-thioguanine associated nodular regenerative hyperplasia in patients with inflammatory bowel disease may induce portal hypertension. Am J Gastroenterol 2007;102:2495-2503.

Reprint requests

Address requests for reprints to: Melek Simsek, MD, VU University Medical Center, Department of Gastroenterology, Room PK 2X 136, De Boelelaan 1118, 1081 HZ Amsterdam, The Netherlands. e-mail: m.simsek@vumc.nl; fax: +31204440554
Conflicts of interest

These authors disclose the following: Annemarie C. de Vries has served as a speaker, member of advisory board, or consultant for Takeda, AbbVie, Tramedico, Dr Falk, and Jansen. Frank Hoentjen has served as a speaker, member of advisory board, or consultant for MSD, Takeda, Celltrion, Teva, Sandoz, Dr Falk, and Celgene. Gerard Dijkstra has received unrestricted research grants from AbbVie and Takeda; has served as a member of the advisory board for Mundipharma and Pharmacosmos; and has received speaker fees from Takeda and Janssen. Jeroen M. Jansen has served as a speaker, member of advisory board, or consultant for AbbVie, Janssen, Ferring, Pfizer, Takeda, and MSD. Bas Oldenburg has served as a speaker, member of advisory board, or consultant for AbbVie, Ferring, Janssen, MSD, Pfizer, and Takeda. Nanne K. H. de Boer has served as a speaker for AbbVie, Takeda, and MSD; has served as consultant and principal investigator for Takeda and TEVA Pharma BV; and has received research grants from Dr. Falk and Takeda. Chris J. J. Mulder has served a principal investigator for TEVA Pharma BV. The remaining authors disclose no conflicts. 